Historic, Archive Document

Do not assume content reflects current scientific knowledge, policies, or practices. 

62.01

$\bullet \bullet \bullet \bullet \bullet \bullet \bullet \bullet \bullet \bullet \bullet \bullet \bullet \bullet \bullet \bullet \bullet \bullet \bullet \bullet \bullet \bullet \bullet \bullet \bullet \bullet \bullet \bullet$

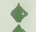

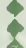

8

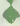

8

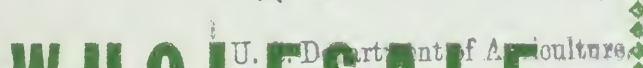
$\therefore 1131131932$ से 11010

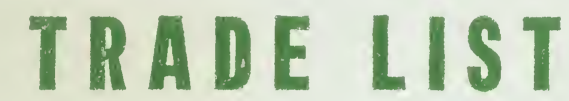

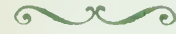

FALL 1932

SPRING 1933

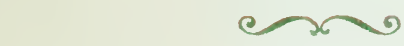

\section{HOWELL NURSERIES}

KNOXYILLE, TENNESSEE

and SWEETWATER, TENNESSEE 


\section{WHOLESALE \\ TRADE LIST}

\section{FALL 1932 \\ SPRING 1933}

\section{HOWELL NURSERIES}

KNOXVILLE, TENN.

The following list constitutes our surplus. In offering it we do so with confidence that we can please, both in quality of stock and in digging and packing.

TERMS: Our terms are net cash or satisfactory reference before shipment. If satisfactory references are given the terms are thirty days.

PRICES: Pric as apply to wholesale quantities and are based on 100 rates.

GUARANTEE: We make no guarantee of any kind whatever, either expressed or implied. All claims, to receive our attention, must be made when the goods are receivd.

SHIPPING AND BOXING: Boxing charged at cost unless cash accompanies order, in which case boxing is free. Explicit shipning instructions should be given in every case.

We wish to thank our patrons for the liberal share of their business given us in the past.

\section{HOWELL NURSERIES}

Knoxville, Tennessee

and Sweetwater, Tennessee 


\section{WHOLESALE LIST}

\begin{tabular}{|c|c|c|}
\hline Abelia grandiflora B \& B & $3-4^{\prime}$ & .45 \\
\hline " " Bare Root & $3-4$ & .35 \\
\hline Acer platanoides-Norway Maple & $6-8$ & .75 \\
\hline 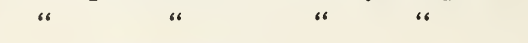 & $8-10$ & 1.00 \\
\hline schwedleri & $5-6$ & 1.00 \\
\hline “ & $6-8$ & 1.25 \\
\hline saccharinum-Silver Maple & $8-10^{\prime}$ & .50 \\
\hline " $\quad$ " & $10-12$ & .75 \\
\hline pyramidalis & $6-8$ & 1.50 \\
\hline “ “ $\quad$ Sugar Maple & $6-8^{\prime}$ & 1.00 \\
\hline Aesculus pavia-Dwarf Buckeye & $12-18^{\prime}$ & .35 \\
\hline Aesculus pavia-Dwarf Buckeye & $12-18^{\prime}$ & .25 \\
\hline Albizzia-Mimosa & $3-4$ & .35 \\
\hline Amorpha fruticosa & $5-6$ & .30 \\
\hline Aralia spinesa & $2-3$ & .20 \\
\hline Aronia arbutifolia & $2-3$ & .20 \\
\hline Azalea hinodigiri $\mathrm{B} \& \mathrm{~B}$ & $8-12 ”$ & .50 \\
\hline " $" \quad$ B \& B & $12-15^{\prime \prime}$ & .75 \\
\hline B \& B & $15-18^{\prime \prime}$ & 1.00 \\
\hline B \& B & $18-21^{\prime \prime}$ & 1.25 \\
\hline
\end{tabular}

All of our Azaleas are extra nice and will give satisfaction. We have an assortment of Azaleas in other varieties mostly in the .75 and $\$ 1.00$ sizes.

Berberis atropurpurea

$2-3$

Add $.15 \mathrm{c}$ if $\mathrm{B}$. \& $\mathrm{B}$

Berberis wilsonae B \& B

$$
\text { " " B \& B }
$$

$18-24 " \quad .75$

$2-3, \quad 1.00$

Betula alba-White Birch

$5-6, \quad .50$

"، “ ،

$8-10^{\prime} \quad 1.00$

Buddleia-Butterfly bush

$3-4^{\prime} \quad .15$

“ alternifolia

18-24" . 15

“ lindleyana

Buxus sempervirens

18-24" .15

$12-18$ " $\quad 1.00$

$18-24 " \quad 1.25$

$2-3$ ' $\quad .20$

Callicarpa americana

$3-4^{\prime} \quad .25$

6"

66

$18-24^{\prime \prime} \quad .15$

Calycanthus floridus

2-3, $\quad .20$

$2-3, \quad .25$

Castanea mollissima-Blight Proof

Chestnut

4-5,

Cedrus deodara B \& B

2-3' $\quad 1.00$

$3-4^{\prime} \quad 1.25$

4-5, $\quad 1.50$

5- ${ }^{\prime}, \quad 2.00$

“ “

$6-S^{\prime} \quad 2.50$ 
Howell Nurseries

\begin{tabular}{|c|c|c|}
\hline & $\begin{array}{r}\text { Size } \\
2-3,\end{array}$ & $\begin{array}{r}\text { Price } \\
1.25\end{array}$ \\
\hline $\begin{array}{ccccc}\text { Cedrus libani } & \text { B } & \& & B \\
\text { " } & * & \text { B } & \& & \text { B }\end{array}$ & $3-4$ & 1.50 \\
\hline $\begin{array}{c}\text { Cephalotaxus harringtonia } \\
\text { fastigiata B \& B }\end{array}$ & $12-18^{\prime \prime}$ & 1.50 \\
\hline $\begin{aligned} \text { Cephalotaxus harringtonia } \\
\text { fastigiata } \mathrm{B} \& \mathrm{~B}\end{aligned}$ & $18-24 "$ & 2.00 \\
\hline Cercidophyllum japonicum & $3-4^{\prime}$ & .60 \\
\hline “ $\quad$ “ & $4-5$, & .75 \\
\hline Cercis canadensis-Red Bud & $6-8$ & .50 \\
\hline “ chinensis & $5-6$ & .50 \\
\hline Chionanthus virginica & $2-3$ & .50 \\
\hline Clethra alnifolia & $18-24 "$ & .25 \\
\hline Cornus florida $\mathrm{B} \& \mathrm{~B}$ & $3-4$ & .75 \\
\hline “ “ $\quad$ plena B \& B & $3-4^{\prime}$ & 1.50 \\
\hline “ rubra B \& B & $2-3$ & .65 \\
\hline “ $\mathrm{B} \& \mathrm{~B}$ & $3-4$ & .90 \\
\hline " “ $\quad$ " $\quad$ B \& $\quad$ B & $4-5$ & 1.35 \\
\hline “ welchi B \& B & $18-24^{\prime \prime}$ & 1.00 \\
\hline Catoneaster divaricata B \& B & $12-18^{\prime \prime}$ & .50 \\
\hline Cotoneaster horizontalis B \& B & $2-3^{\prime}$ & .60 \\
\hline “ $\quad$ “ $\quad$ B \& B & $3-4^{\prime}$ & .75 \\
\hline Cydonia japonica & $18-24 "$ & .20 \\
\hline Deutzia gracilis rosea & $2-3$ & .15 \\
\hline Eleagnus reflexa $B$ \& $B$ & $3-4^{\prime}$ & .75 \\
\hline “ $\quad$ “ broad leaf B \& B & $3-4^{\prime}$ & 1.00 \\
\hline Euonymus alatus & $3-4$ & .50 \\
\hline “ americanus & $2-3$ & .20 \\
\hline “ radicans $\mathrm{B} \& \mathrm{~B}$ & $4 \mathrm{yr}$. & .25 \\
\hline “ $\quad$ staked B \& B & $4 \mathrm{yr}$. & .55 \\
\hline “ seiboldi patens B \& B & $12-18 "$ & .40 \\
\hline $\begin{array}{lllllll} & \cdots & \cdots & \cdots & B & \& & B\end{array}$ & $2-3$ & .60 \\
\hline Exochorda grandiflora & $2-3$ & .20 \\
\hline Forsythia fortunei & $3-4^{\prime}$ & .20 \\
\hline “ seiboldi & $3-4$ & .20 \\
\hline spectabilis & $12-18^{\prime \prime}$ & .10 \\
\hline suspensa & $2-3$ & .15 \\
\hline “ & $3-4^{\prime}$ & .20 \\
\hline viridissima & $2-3$ & .15 \\
\hline Halesia tetraptera & $18-24 "$ & .20 \\
\hline “ & $2-3$ & .25 \\
\hline “ & $3-4^{\prime}$ & .30 \\
\hline Hamamelis virginica & $2-3^{\prime}$ & .20 \\
\hline “ & $5-6$ & .35 \\
\hline “ $\quad$ “ & $6-8^{\prime}$ & .50 \\
\hline $\begin{array}{c}\text { Hesperaloe parvifolia-Red Yucca } \\
\text { “ }\end{array}$ & $\begin{array}{ll}2 & \mathrm{yr} \\
4 & \mathrm{yr}\end{array}$ & $\begin{array}{l}.25 \\
.50\end{array}$ \\
\hline Hydangea Hills of Snow & $18-24 "$ & .15 \\
\hline “ & $2-3$ & .20 \\
\hline paniculata grandiflora & $2-3$ & .20 \\
\hline
\end{tabular}




\section{Hypericum calycinium \\ moserianum}

Ilex crenata B \& B

“ “ B \& B

" " " $\quad$ \& B

" " B \& B

" cornuta B \& B

" decidua

"،

“ opaca B \& B

“ " B \& B

“ " grafted B \& B

“ vomitoria B \& B

" " B \& B

" "

Jasminum nudiflorum

Juniperus chinensis albo-variegata (shuki) B \& B

Juniperus chinensis albo-variegata B \& B

Juniperus chinensis albo-variegata B \& B

Juniperus chinensis densa glauca B \& B

Juniperus chinensis densa glauca B \& B

Juniperus chicensis japonica

B \& B

Juniperus chi:ensis japonica B \& B

Juniperus chi ensis japonica sylvestris B \& B

Juniperus chizensis japonica

sylvestris B \& B

$\begin{array}{llllll}\text { " “ } & \text { mascula B \& B } & 2-3 & & 1.00 \\ \text { " } & \text { " } & \text { B \& B } & 3-4, & 1.25\end{array}$

Juniperus chinenesis pfitzeriana

B \& B

Juniperus chinensis pfitzeriana

$\mathrm{B} \& \mathrm{~B}$

Juniperus chinenesis pfitzeriana B \& B

Juniperus chinensis pfitzeriana

B \& B

Juniperus chinenesis pfitzeriana

B \& B

Juniperus chi-ensis procumbens

B \& B

$12-18 " \quad .60$

18-24” . .75

$2-3^{\prime} \quad 1.00$

$3-4^{\prime} \quad 1.25$

$4-5^{\prime} \quad 1.50$

12-18" $\quad 1.00$

Juniperus communis ashfordi B \& B $2-3$ ' $\quad .75$
66
66
"6
B \& B 3-4'
1.00 


\begin{tabular}{|c|c|c|}
\hline & Size & Price \\
\hline $\begin{array}{l}\text { Juniperus communis depressa } \\
\text { aurea B \& B }\end{array}$ & 18-24" & .75 \\
\hline $\begin{array}{l}\text { Juniperus communis depressa } \\
\text { aurea B \& B } \\
\text { Juniperus communis depressa }\end{array}$ & $2-3$ & 1.00 \\
\hline nana $B$ \& $B$ & $18-24 "$ & .75 \\
\hline $\begin{array}{c}\text { Juniperus communis depressa } \\
\text { nana B \& B }\end{array}$ & $2-21 / 2$ & 1.00 \\
\hline $\begin{array}{l}\text { Juniperus communis depressa } \\
\text { nana B \& B }\end{array}$ & $21 / 2-3$ & 1.25 \\
\hline $\begin{array}{l}\text { Juniperus communis depressa } \\
\text { plumosa B \& B }\end{array}$ & $12-18^{\prime \prime}$ & .75 \\
\hline $\begin{array}{l}\text { Juniperus communis depressa } \\
\text { plumosa B \&B }\end{array}$ & $18-24 "$ & .85 \\
\hline $\begin{array}{l}\text { Juniperus communis depressa } \\
\text { plumosa B \& B }\end{array}$ & $2-3$ & 1.00 \\
\hline Juniperus chinenesis hibernica B \& B & $2-3$ & .50 \\
\hline " $\quad$ " $\quad$ " $\quad$ " $\quad$ B \& B & $3-4^{\prime}$ & .75 \\
\hline " $\quad$ “ $\quad$ B \& $\quad$ B & $4-5$ & 1.00 \\
\hline $\begin{array}{llllll}\cdots & \cdots & \text { B } & \& & \text { B }\end{array}$ & $5-6$ & 2.00 \\
\hline $\begin{array}{l}\text { Juniperus chinensis oblonga pendula } \\
\text { B \& B }\end{array}$ & $2-3$ & .50 \\
\hline Juniperus chinensis suecica B \& B & $2-3$ & .75 \\
\hline “ kesteriana B \& B & $4-5$ & 1.50 \\
\hline " naeboriensis $B$ \& $B$ & $3-4^{\prime}$ & 1.25 \\
\hline “ sabina $B$ \& $B$ & $18-24^{\prime \prime}$ & .50 \\
\hline " $\quad$ " B \& B & $2-3$ & .60 \\
\hline Kolkwitzia amabilis & $18-24 "$ & .35 \\
\hline " & $2-3$ & .40 \\
\hline “ & $3-4$ & .50 \\
\hline “ & $4-5$, & .60 \\
\hline $\begin{array}{c}\text { Lagerstroemia Wm. Toovey } \\
\text { “ }\end{array}$ & $\begin{array}{l}2-3 \\
3-4\end{array}$ & $\begin{array}{l}.30 \\
.40\end{array}$ \\
\hline Laurocerasus officinalis $B \& B$ & $2-3$ & .75 \\
\hline " $\quad$ " $\quad$ B \& B & $3-4^{\prime}$ & 1.00 \\
\hline Libocedrus decurrens B \& B & $4-5$ & 2.00 \\
\hline “ $\quad$ “ $\quad$ B \& B & $5-6$ & 3.00 \\
\hline Ligustrum lodense & $6-12 "$ & .15 \\
\hline “ lucidum $B$ \& $B$ & $2-3$ & .75 \\
\hline pendulum & $18-24^{\prime \prime}$ & .20 \\
\hline “ & $3-4^{\prime}$ & .25 \\
\hline quihoui & $4-5$ & .35 \\
\hline " sinense-Amur River South & $2-3$ & .02 \\
\hline Lonicera fragrantissima & $2-3$ & .15 \\
\hline “ & $5-6$ & .35 \\
\hline heckrotti & $18-25 "$ & .15 \\
\hline morowi & $3-4$ & .25 \\
\hline nitida $B$ \& $B$ & $2^{\prime}$ & .50 \\
\hline species-best berry bearers & $2-3$ & .20 \\
\hline tatarica & $3-4^{\prime}$ & .25 \\
\hline thibetica & $2-3$ & .25 \\
\hline
\end{tabular}


Magnolia glauca B \& $\mathrm{B}$

Size

Price

$$
\text { “ “ B \& B }
$$

$2-3$

.75

“ grandiflora B \& B

$3-4, \quad 1.00$

" " B \& B

$2-3, \quad .60$

Magnolia purpurea-soulangeana

nigra $B$ \& $B$

3-4'

Magıolia purpurea-soulangeana nigra $B$ \& $B$

$18-24 " \quad 1.00$

$2-3, \quad 1.50$

Magnolia purpurea-soulangeana nigra $B \quad \& B$

$3-4^{\prime} \quad 2.00$

“ stellata B \& B

18-24" 2.00

“ “ B \& B

$2-3, \quad 3.00$

“ tripetala

، "

$4-5, \quad .50$

5ं 6 , $\quad .75$

“ “

$6-8^{\prime} \quad 1.00$

Mahonia aquifolium B \& B

$12-18 " \quad .40$

" " $\quad$ B \& B

18-24" . .50

“. " $\quad$ B \& B

Na:-dina domestica B \& B

$2-3, \quad .75$

Nandina domestica B \& B

Osmanthus aquifolium B \& B

18-24" .50

$2-3$, $\quad .60$

Philadelphus coquette

$12-18 " \quad 1.25$

Photinia serrulata B \& B

18-24" . .15

$$
\text { " " B \& B }
$$

Picea excelsa B \& B

2-3' $\quad 1.00$

$3-4, \quad 1.25$

$2-3, \quad .75$

“ pungens $\mathrm{B} \& \mathrm{~B}$

$18-24 ", \quad 1.00$

Pinus montana mughus $\mathrm{B}$ \& $\mathrm{B}$

12-18" .75

“ strobus B \& B

2-3' $\quad 1.00$

" " B \& B

$3-4^{\prime} \quad 1.25$

" " "B \& B

$4-5, \quad 1.50$

Platanus occidentalis

$6-8^{\prime} \quad .60$

$8-10, \quad 1.00$

Prunus tomentosa

2-3' $\quad .35$

2-3, $\quad 1.00$

Pyracantha lalandi B \& B

Retinospora ericoides B \& B

$18-24 " \quad .50$

" “ B \& B

$2-3$, $\quad .60$

“ filifera B \& B

" " $\mathrm{B} \& \mathrm{~B}$

$2-3, \quad 1.00$

3-4' $\quad 1.50$

“ " $\quad$ B \& B

$4-5, \quad 2.50$

Retinospora obtusa aurea youngi B \& B

$3-4^{\prime} \quad 1.50$

Retinospora obtusa aurea youngi

$\mathrm{B} \& \mathrm{~B}$

$4-5, \quad 2.00$

$2-3, \quad 2.00$

Retinospora obtusa nana B \& B

$18-24$ " .75

“ pisifera B \& B

5-6, $\quad 1.50$

“ aurea B \& B

$6-8, \quad 2.50$ 


\begin{tabular}{|c|c|c|}
\hline & & \\
\hline Retinospora plumosa B \& B & $3-4^{\prime}$ & 1.00 \\
\hline “ $\quad$ “ $\quad$ B \& B & $4-5^{\prime}$ & 1.50 \\
\hline $\mathrm{B} \& \mathrm{~B}$ & $5-6$ & 2.00 \\
\hline $\mathrm{B} \& \mathrm{~B}$ & $6-8$ & 2.50 \\
\hline aurea B \& B & $3-4^{\prime}$ & 1.00 \\
\hline “ $\quad \begin{array}{lllll} & \text { B } & \& & B\end{array}$ & $4-5$ & 1.25 \\
\hline $\begin{array}{lllll} & \cdots & \text { B } & \& & B\end{array}$ & $5-6$ & 2.00 \\
\hline Rhodotypos kerroides & $2-3$ & .20 \\
\hline Rhus aromatica & $18-24^{\prime \prime}$ & .15 \\
\hline " 6 & $3-4^{\prime}$ & .25 \\
\hline cotonoides-American Smoketree & $2-3$ & .30 \\
\hline $\begin{array}{lll}* & \cdots & \cdots\end{array}$ & $3-4^{\prime}$ & .40 \\
\hline " cotinus-Purple Fringe & $2-3$ & .30 \\
\hline Rosa rugosa & $2-3$ & .20 \\
\hline Salix caprea-Pussy Willaw & $4-5$ & .40 \\
\hline Senecio-Groundsel Tree & $4-5^{\prime}$ & .50 \\
\hline Spiraea Anthony Waterer & $12-18^{\prime \prime}$ & .15 \\
\hline “ “ “ “ heavy & $18-24^{\prime \prime}$ & .20 \\
\hline “ opulifolia & $3-4^{\prime}$ & .25 \\
\hline “ prunifolia & $3-4^{\prime}$ & .25 \\
\hline “ thunbergi heavy & $3 \mathrm{ft}$. & .20 \\
\hline " trichocarpa & $2-3$ & .25 \\
\hline “ van houttei heavy & $3-4$ & .20 \\
\hline Sterculea platinifolia & $4-5$ & .75 \\
\hline Styrax americana & $2-3$ & .30 \\
\hline “ japonica & $18-24^{\prime \prime}$ & .25 \\
\hline Symphoricarpos chenaulti & $4-5^{\prime}$ & .20 \\
\hline “ racemosus & $2-3$ & .20 \\
\hline ‘ & $3-4^{\prime}$ & .25 \\
\hline “ vulgaris & $2-3$ & .12 \\
\hline Taxodium distichum & $2-3$ & .35 \\
\hline Taxus canadensis aurea B \&B & $12-18^{\prime \prime}$ & 1.00 \\
\hline Thea bohea $B$ \& $B$ & $18-24 "$ & .60 \\
\hline Thuya occidentalis $B$ \& $B$ & $2-3$ & .50 \\
\hline $\begin{array}{lllll} & \cdots & \text { B \& } & \text { B }\end{array}$ & $3-4^{\prime}$ & .75 \\
\hline $\mathrm{B} \& \mathrm{~B}$ & $4-5$ & 1.25 \\
\hline$B \& B$ & $5-6$ & 1.50 \\
\hline
\end{tabular}

Thuya occidentalis ellwargeriana

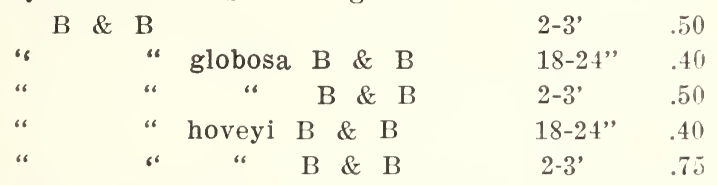

Thuya occidentakis lutea or George

$$
\text { Peabody B \& B 2-3’ } 1.00
$$

Thuya occidentalis lutea or George

Peabody B \& B $3-4^{\prime} \quad 1.25$

Thuya occidentalis plicata pyramidalis

B \& B 3-4” 1.25

Thuya occidentalis plicata pyramidalis

B \& B '4-5’ 1.50 


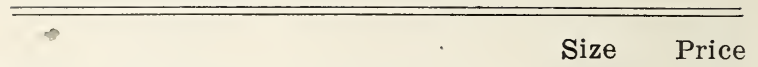

Thuya occidentalis pumila B \& B 18-24" $\quad .50$ 'Thuya occidentalis pyramidals B \& B 2-3' .75

\begin{tabular}{|c|c|c|c|c|c|}
\hline “ & “ & “ & B \& & $3-4^{\prime}$ & 1.25 \\
\hline “ & “ & ‘، & B \& & $4-5$ & 1.50 \\
\hline ، & “" & “ & B \& & $5-6$ & 2.00 \\
\hline & “ & “ & B \& & $6-8$ & 3.00 \\
\hline & 6 & reidi $B$ \& $B$ & & $18-24^{\prime \prime}$ & .75 \\
\hline & “ & “ B \& B & & $2-3$ & 1.00 \\
\hline & “" & wareana B \& & B & $18-24 "$ & .75 \\
\hline
\end{tabular}

Thuya orientalis aurea conspicua

$$
\begin{array}{llll}
\text { B \& B } \quad 3-4^{\prime} & 1.00
\end{array}
$$

Thuya orientalis aurea conspicua $4-5$, 1.25

Thuya orientalis aurea nana B \& B $18-24$ " $\quad .50$

“ “ “ “ “ B \& B $\quad 2-3$ ” $\quad .60$

" “ " " bakeri B \& B 3-4” 1.00

“ “ pyramidalis green B \& B 6-8” 3.50

Tsuga canadensis-Hemlock B \& B 18-24" .75

“ “ “ “ $\quad$ B \& B $2-3$ ' 1.00

" " “ “ 4 B \& B $3-4$ " 1.50

" “ “ $4 \quad$ B \& B $4-5, \quad 2.00$

" “ “

specimens B \& B 2-3’ 1.25

Tsuga canadensis-Hemlock Sheared specimens B \& B $3-4^{\prime} \quad 2.00$

Tsuga canadensis-Hemlock Sheared $\begin{array}{lll}\text { specimens B \& B } & 4-5^{\prime} & 2.50\end{array}$

Tsuga canadensis-Hemlock Sheared $\begin{array}{lll}\text { specimens B \& B } & 5-6, & 3.50\end{array}$

Ulmus americana $\quad 8-10^{\prime}, \quad .75$

“ “ $10-12$ ' 1.25

“ chinensis $5-6, \quad .50$

Viburnum carlesi B \& B 2-3' 1.50

\begin{tabular}{|c|c|c|c|}
\hline “ & lantana & $3-4$ & .35 \\
\hline “" & opulus & $2-3$ &. .15 \\
\hline “ & “ sterilis & $18-24 "$ & .12 \\
\hline “ & " $" ~ “$ & $3-4^{\prime}$ & .20 \\
\hline “ & rytidophyllum B \& B & $2-3^{\prime}$ & 1.00 \\
\hline ‘6 & “ $\quad$ B \& B & $3-4^{\prime}$ & 1.25 \\
\hline ‘ & tomentosum & $2-3$ & .20 \\
\hline & “ plicatum & $2-3$ & .30 \\
\hline & entosa & 4 yr. & .25 \\
\hline
\end{tabular}

Yucca filimentosa $\quad 4$ yr. $\quad .25$ 
